\title{
Bacteremia in adult cancer patients with apparently stable febrile neutropenia: data from a cohort of 692 consecutive episodes from a single institution
}

\author{
Alberto Carmona-Bayonas, $\mathrm{MD}, \mathrm{PhD},{ }^{\mathrm{a}}$ Paula Jiménez Fonseca, $\mathrm{MD}, \mathrm{PhD},{ }^{\mathrm{b}}$ Carme Font, \\ $\mathrm{MD}, \mathrm{PhD},{ }^{\mathrm{c}}$ Jerónimo Martínez-García, MD, $\mathrm{PhD},{ }^{\mathrm{d}}$ Francisco Torrella, $\mathrm{MD}, \mathrm{PhD},{ }^{\mathrm{a}}$ Edgar \\ Urrego, MD, PhD, ${ }^{a}$ Patricia Castaño, MD, PhD, ${ }^{a}$ Agustín Navarrete, MD, PhD, ${ }^{\mathrm{d}}$ Maite \\ Antonio-Rebollo, MD, PhD, ${ }^{\mathrm{a}}$ Joaquín Gómez Gómez, MD, PhD,${ }^{\mathrm{e}}$ Vicente Vicente, ${ }^{\mathrm{a}}$ \\ Francisco Ayala, MD, $\mathrm{PhD}^{\mathrm{a}}$
}

Departments of ${ }^{a}$ Hematology and Medical Oncology, Hospital Morales Meseguer, Murcia; ${ }^{b}$ Medical Oncology, Hospital Central de Asturias, Oviedo; ${ }^{\mathrm{c} M e d i c a l}$ Oncology, Hospital Clinic, Barcelona; ${ }^{\mathrm{d} M e d i c a l}$ Oncology, Hospital Virgen de la Arrixaca, Murcia; and 'Infectious Diseases, Hospital Virgen de la Arrixaca, Murcia, Spain

Background Bacteremia is associated with increased risk of complications in patients with febrile neutropenia (FN), although few clinical studies have reported outcomes in apparently stable patients (ASPs) who could be candidates for home treatment.

Objective To assess the risk factors and the impact of bacteremia in ASPs.

Methods We retrospectively analyzed 861 consecutive episodes of FN that were classified according to their presentation into 2 categories: clearly unstable patients and ASPs. We estimated the incidence of bacteremia and severe complications in ASPs. We analyzed predictors for bacteremia and the discriminatory ability of the MASCC score in this setting.

Results We classified 692 episodes as ASPs. Bacteremia occurred in 6\%, major complications were noted in $7.3 \%$, and death occurred in $1.3 \%$. Patients with bacteremia had more complications (odds ratio [OR], 8.2), and mortality (OR, 8.2). The integration of the MASCC score and bacteremic status predicted complications with an area under the receiver operating characteristic $(\mathrm{ROC})$ curve of 0.74 , sensitivity of $36 \%$, and specificity of $94 \%$. Predictors of bacteremia were temperature $\geq 39^{\circ} \mathrm{C} / 102.2^{\circ} \mathrm{F}(\mathrm{OR}$ $3)$, rigors $(O R, 2.2)$, ECOG PS $\geq 2(O R, 2.1)$, and advanced cancer $(O R, 2.5)$. Two percent of patients who remained afebrile for 48 hours had positive blood cultures afterward.

Limitations A single-center, retrospective analysis, and the absence of a validation set to test the model's discriminatory ability. Conclusions Bacteremia is infrequent among ASPs but is associated with a high risk of complications. We identified several variables that could improve the prognostic classification of clinically stable FN.

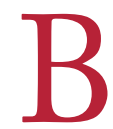
acteremia is associated with adverse outcomes, including serious complications and increased mortality, in patients with febrile neutropenia (FN). ${ }^{1-3}$ Therefore, early recognition of these cases is important because inappropriate initial therapy is related to an increase in overall mortality. 4,5 According to current evidence-based guidelines, oral ambulatory treatment is an alternative in low-risk patients. ${ }^{6}$ The detection of bacteremia is relatively infrequent in this scenario, because the pretest probability and blood culture yield are expected to decrease. ${ }^{7}$ Thus, bacteremia is reported in $10 \%-30 \%$ of $\mathrm{FN}$ episodes, ${ }^{8}$ with a lower prevalence (7\%-10\%) in low-risk groups. ${ }^{9,10}$ Nonetheless, the detection of micro-organisms usually requires $24-48$ hours, ${ }^{11}$ resulting in some patients being discharged from hospital, which can lead to a cause of treatment failure. ${ }^{12}$ On laboratory determination of bacteremia, reassessment of the clinical condition, microbial sensitivity, and treatment adherence are necessary, ${ }^{13}$ with a proportion of these patients requiring intravenous therapy. ${ }^{14,15}$

The MASCC (Multinational Association for Supportive Care in Cancer) index is the most widely accepted model to identify low-risk episodes of $\mathrm{FN}$ that can be safely treated at home. ${ }^{16}$ Nonetheless, it remains under debate whether bacteremic patients with predicted low-risk episodes could benefit from hospital admission and

Accepted for publication June 30, 2014. Correspondence: Alberto Carmona-Bayonas; alberto.carmonabayonas@gmail. com. Disclosures: The authors have disclosed no conflicts of interest. JCSO 2014;12:312-320. (C2014 Frontline Medical Communications. DOI 10.12788/jicso.0071 
aggressive intravenous antibiotic therapy while they wait for the blood culture results. ${ }^{17,18}$ Hence, it would be of great clinical interest to identify low-risk FN patients who present with bacteremia so that outcomes can be optimized.

We have performed a nested case-control study to determine the prognostic impact of bacteremia in this population. A secondary objective was to analyze the risk factors for bacteremia in clinically stable outpatients.

\section{Methods}

\section{Patients}

We reviewed the medical records of all adult ( $\geq 18$ years) outpatients who were admitted to an oncology ward in a tertiary-care hospital during 1998-2006 with a diagnosis of FN within the setting of a solid neoplasm. FN was defined as fever $\geq 38{ }^{\circ} \mathrm{C}$ for over $1 \mathrm{~h}$, with neutropenia $\leq 500$ cells $/ \mathrm{mm}^{-3}$ (or $\leq 1,000$ cells $/ \mathrm{mm}^{-3}$ but with a predicted decrease to $\leq 500$ cells $/ \mathrm{mm}^{-3}$ ). Episodes of $\mathrm{FN}$ were excluded if the work-up and antibiotic therapy did not comply with contemporary Infectious Diseases Society of America guidelines. ${ }^{19}$ The study was approved by the institution's local ethics committee.

\section{Study design}

Patients were classified into 2 main categories: clearly unstable patients (CUPs) and apparently stable patients (ASPs), as previously defined by our group. ${ }^{20}$ CUPs were defined as cases with acute septic shock, hypotension, acute organ failure, and/or extensive infection (meningitis, pneumonia, typhlitis, cholangitis, pyelonephritis, or cellulitis $>5 \mathrm{~cm}$ in diameter and were excluded from analysis because they represented well-known and easily recognizable high-risk situations. Patients diagnosed with FN but with none of the aforementioned criteria were considered to be ASPs and were included in the study. During the initial work-up, 2 sets of blood cultures were obtained before antimicrobial therapy, with samples drawn through each lumen of a central venous catheter, if present, or from 2 different peripheral vein sites.

\section{Outcomes and predictive factors for bacteremia}

The main outcome measure was the incidence of clinically significant bacteremia in ASPs with FN. All of these cases fulfilled the definition of clinically significant bacteremia in accordance with the US Centers for Disease Control and Prevention's National Healthcare Safety Network (CDC-NHSN) criteria. $^{21}$ Therefore, isolation of coagulase-negative staphylococci (CoNS) was considered as contamination unless 2 blood samples were positive or in cases of central-line-associated infections.

Other endpoints were: failure of antimicrobial therapy, time of hospitalization, intended duration of the antibiotic treatment, time to defervescence, and serious complications as defined by Klastersky and colleagues ${ }^{7}$ The medical records of the ASP group were analyzed to estimate the incidence of bacteremia and complications. To assess the risk factors for bacteremia in the ASPs, we designed a nested case-control study. Cases were defined as outpatients with clinically relevant bacteremia $(n=42)$. Patients without bacteremia were randomly assigned to the control group with a ratio of $1: 4$ for cases and controls, respectively, and an additional 25\% of controls to compensate for missing values $(n=265)$. On the basis of a review of the literature, we selected the potential predictors for bacteremia. We omitted variables that were not available in the emergency department setting. Time to blood culture positivity was calculated from the time of admission. For continuous variables, cut-offs were defined according to common use and US National Cancer Institute's toxicity scale criteria. A MASCC score of $<21$ was used to define high risk. Clinical stage M1 (TNM IV) and unresected, locally advanced (TNM III) cancer associated with anatomic distortions were defined as advanced cancer.

\section{Statistical considerations}

Standard statistics were used to describe our sample. For univariate analysis, we used the chi-squared and Fisher exact tests to identify the potential risk factors for bacteremia. The Mann-Whitney test was used to compare means. A combination of host features representing chronic vulnerability and acute traits related to microbial virulence were considered (Table 1). All of the tests were two-sided, and $P$ values of $<.05$ were considered significant.

We calculated the sensitivity and specificity of the MASCC (Multinational Association for Supportive Care in Cancer) index to predict both complications and bacteremia. We relied on the area under receiver operating characteristic (ROC) curve to assess the discrimination ability of the MASCC score in this population. To address the problem of integrating bacteremic status and the MASCC score, several models were constructed by means of subtracting points from the MASCC score in bacteremic episodes, with the final combined model being selected as the highest area under the ROC curve.

Univariate and multivariate logistic regression analyses were performed to determine the risk factors for bacteremia. Variables with $P$ values of $<.10$ were eligible for multivariate analysis. Potential overlapping or redundancy was a criterion for exclusion. A bootstrap procedure was used to test the model fit and derive biascorrected confidence intervals. The statistical analyses were carried out with the SPSS 21 software (SPSS Inc, Chicago, IL, USA). 


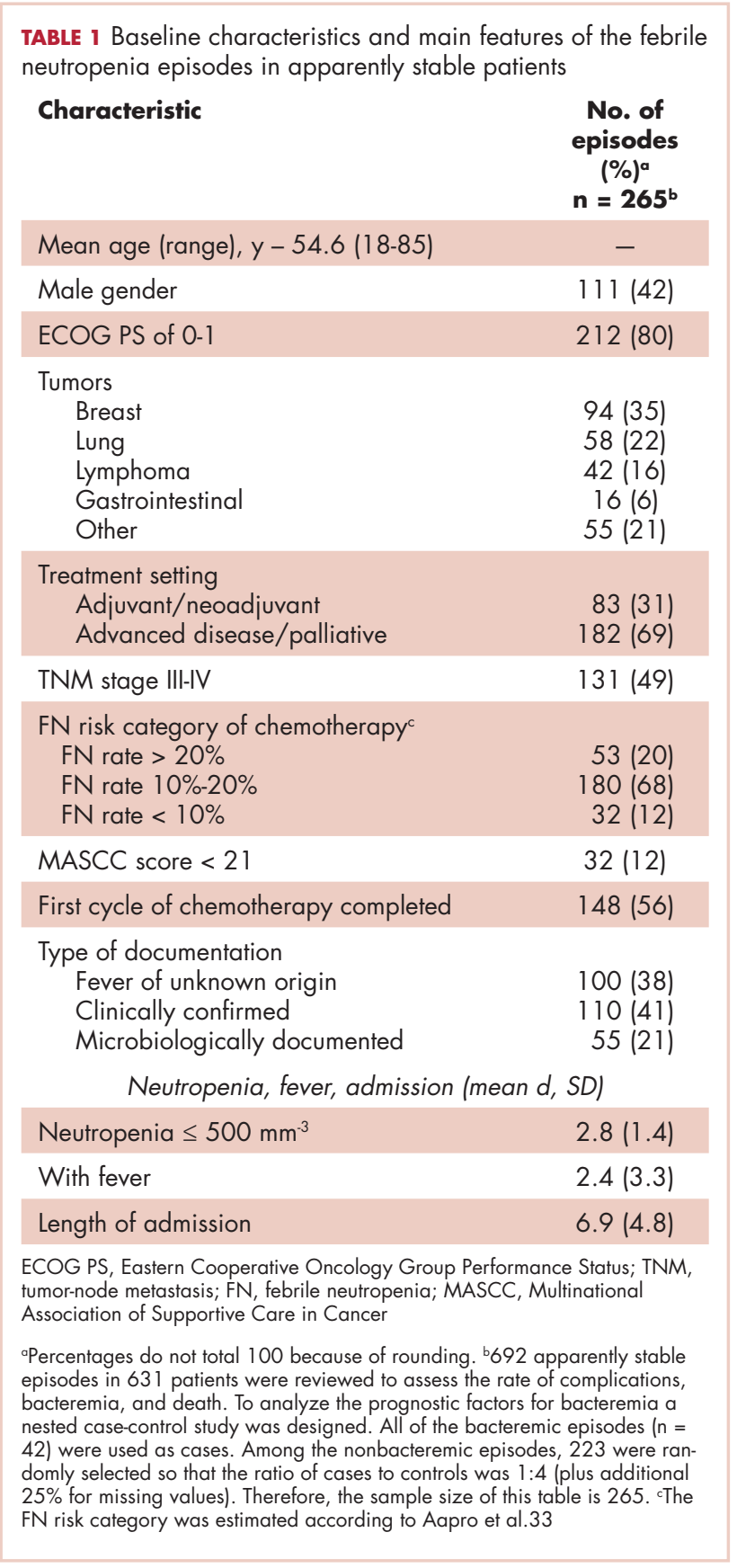

\section{Results}

\section{Patients and febrile neutropenic episodes}

We reviewed the medical records of 861 episodes of FN. Of those, 692 episodes in 631 patients were classified as ASPs (80\%), and 169 (20\%) as CUPs (Figure 1). The baseline characteristics of the ASP group and the main features of the FN episodes are shown in Table 1 . Notably, the rates of bacteremia $(6 \%, n=42)$, serious complications $(7.3 \%$, $\mathrm{n}=51)$, and inpatient mortality $(1.3 \%, \mathrm{n}=9)$ were lower than the average rates reported in other studies. The most

\begin{tabular}{|c|c|}
\hline Isolate & $\begin{array}{l}\text { No. of } \\
\text { episodes (\%) } \\
n=42\end{array}$ \\
\hline Escherichia coli & $13(30)$ \\
\hline Pseudomonas aeruginosa & $9(21)$ \\
\hline Coagulase-negative Staphylococcus & $4(9)$ \\
\hline Staphylococcus aureus & $4(9)$ \\
\hline Streptococcus spp. & $3(7)$ \\
\hline Enterococcus faecalis & $2(5)$ \\
\hline Pseudomonas fluorescens & $1(2)$ \\
\hline Enterobacter cloacae & $1(2)$ \\
\hline Staphylococcus haemolyticus & $1(2)$ \\
\hline Bacteroides uniformis & $1(2)$ \\
\hline Bacillus spp. & $1(2)$ \\
\hline Salmonella typhi & $1(2)$ \\
\hline Klebsiella oxyłoca & $1(2)$ \\
\hline
\end{tabular}

frequent microorganisms isolated in the 42 episodes with bacteremia were Escherichia coli $(30 \%, \mathrm{n}=13)$, followed by Pseudomonas aeruginosa $(21 \%, \mathrm{n}=9)$, CoNS $(9 \%, \mathrm{n}=4)$, Staphylococcus aureus $(9 \%, \mathrm{n}=4)$, and Streptococcus spp. $(7 \%$, $\mathrm{n}=3$; Table 2). In addition, 2 CoNS were not considered as true bacteremias but as potential contaminants. The time to blood culture positivity was less than 24 hours in $16 \%$ of the 42 episodes with bacteremia, $24-48$ hours in $21 \%$, and 48-72 hours or longer in the remaining patients.

Of the total set of blood cultures, $64 \%(n=27)$ were due to Gram-negative bacteria. Among cases with bacteremia, the most common diagnoses were fever without a focus of infection in $61 \%(\mathrm{n}=26)$, moderate infections (urinary and chronic bronchitis) in 21\% ( $n=9)$, mild infections (cellulitis, odontogenic infection, mild enteritis, and upper respiratory infections) in $19 \%(n=8)$ and catheter-related infection in $2 \%(n=1)$.

\section{Outcomes associated with positive blood cultures}

Patients with bacteremia presented with more complications than did the nonbacteremic controls ( $33 \%$ vs $5.6 \%$; odds ratio [OR], 8.2; $P<.0001)$, mainly hypotension and acute respiratory failure. Compared with the controls, patients with bacteremia presented with the following:

A nearly 4-fold more frequent fever lasting more than 24 hours (59\% vs $13 \%, P<.0001)$;

- A longer mean time to defervescence (3.8 vs 2.1 days, $P=.008)$; 


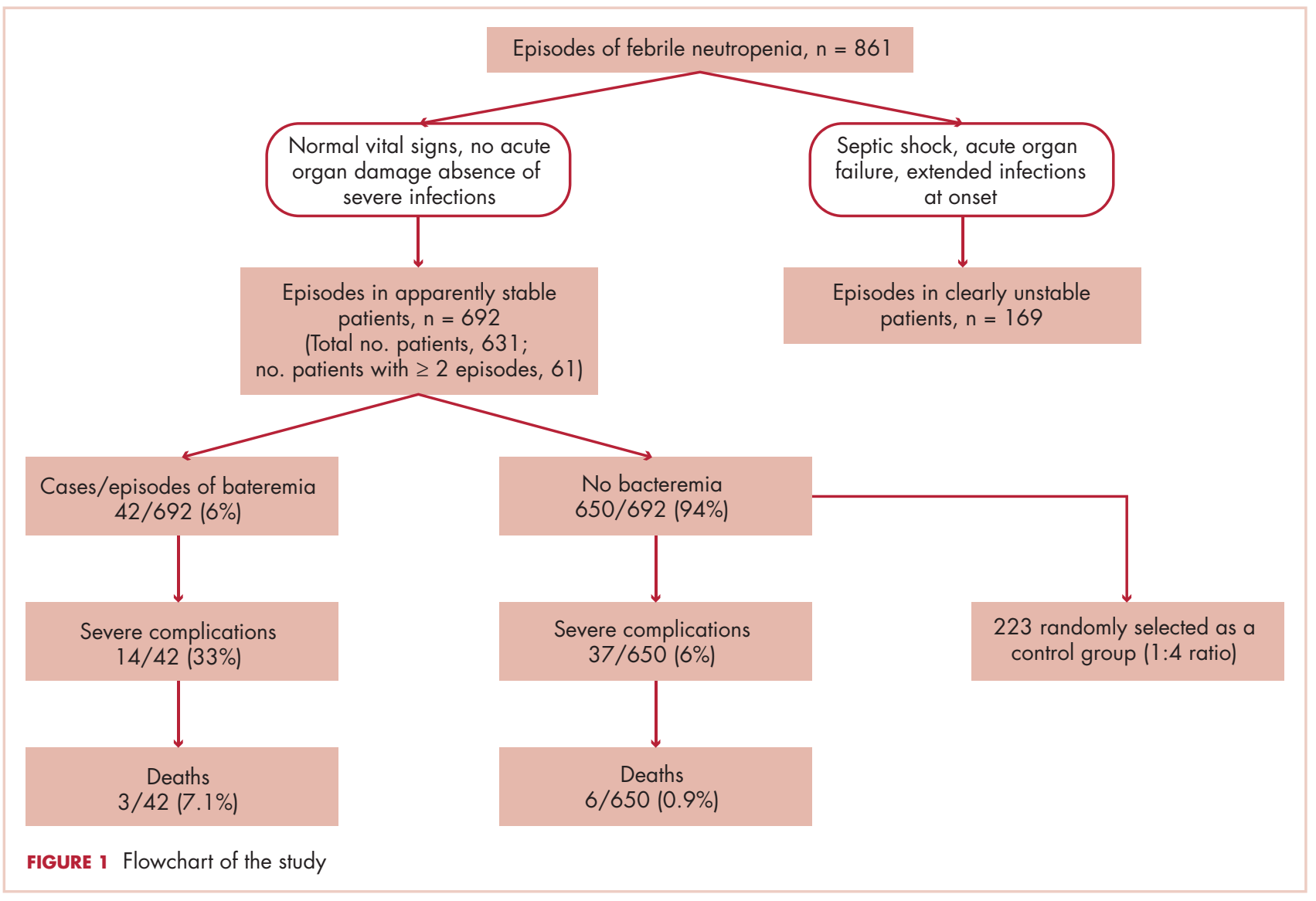

- More frequent treatment failure $(43.9 \%$ vs $8.4 \%$, $P=<.001)$, persisting even on restricting the analysis to either failures due to clinical progression or microbiological criteria ( $18.6 \%$ vs $5 \%, P=.01)$;

- A longer hospital stay (9.9 vs 6.4 days, $P=.001$ );

- Development of delayed lung infiltrates $(9.5 \%$ vs 2.7\%; OR, 3.6; $P=.03)$; and

- Higher overall mortality (7.1\% vs $0.9 \%$; OR, 8.2; $P=.01)$.

On analysis of the prognostic role of other concurrent sites of infection alongside with bacteremia, bacteremic lower respiratory tract infection was the only diagnosis associated with adverse outcomes ( $60 \%$ vs $25 \%, P=.05)$.

\section{Role of the MASCC index for predicting} complications in patients with bacteremia

We observed a significant trend to low-risk episodes (MASCC, $\geq 21$ ) in nonbacteremic patients compared with those with bacteremia (90\% vs $76.2 \%$, respectively; $P=.01)$. The ROC curves obtained on application of the MASCC score, with or without bacteremic status, to the ASP cohort did not significantly differ (Figure 2). The MASCC score was found to detect unexpected complications in the whole ASP group, with the follow-

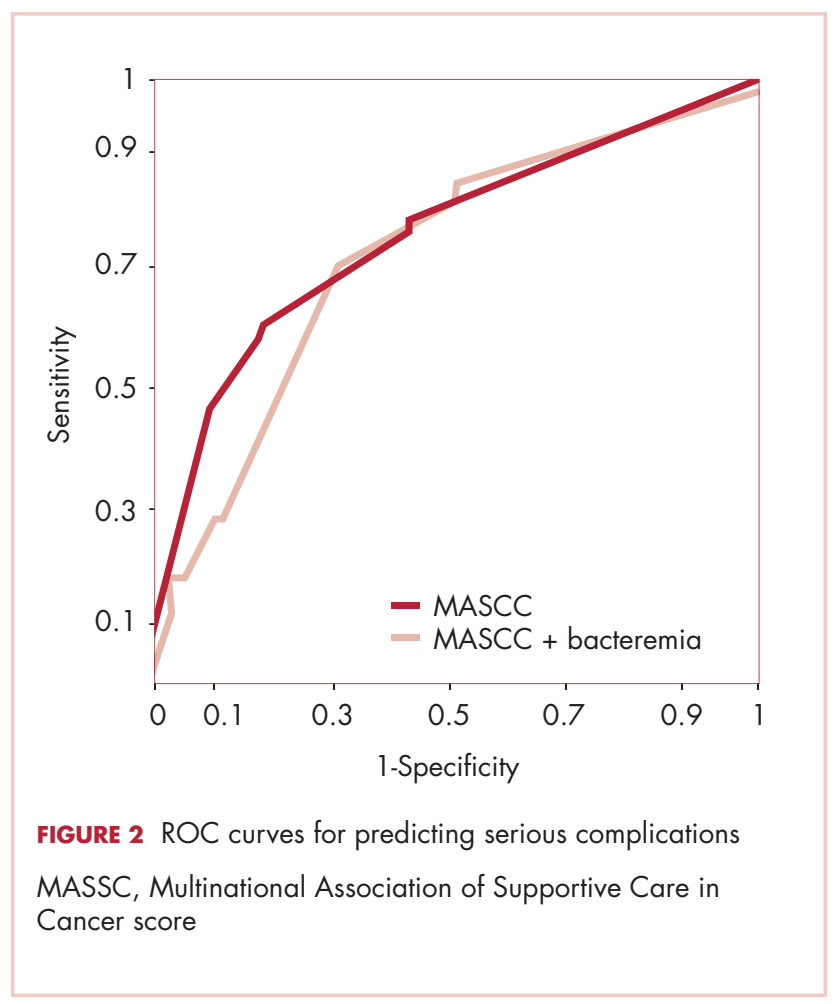




\section{Original Report}

ing predictive values: area under the ROC curve of 0.74 (95\% confidence index [CI], 0.66-0.83), a sensitivity of $36 \%$, and a specificity of $94 \%$. Because bacteremia was relatively infrequent, the overall prognostic effect on the whole ASP group was limited. The combination of the MASCC score and bacteremic status did not increase the discriminatory ability for identifying complications over the MASCC score (area under the ROC curve of 0.75 [95\% CI, 0.69- 0.81]).

As a result of the previous selection of stable patients, no case of hypotension, previous inpatient status or acute leukemia was included, thereby defining a very different sample set from that in which the MASCC score was developed (Table 3). Therefore, on considering only bacteremic patients, the MASCC score was useful, albeit limited to chronic obstructive pulmonary disease (COPD; OR, 4.6) and dehydration (OR, 5), with no statistically significant differences in the remaining covariates. Severe complications were lower in bacteremic patients with predicted low-risk (MASCC, $\geq 21$ ) episodes ( $25 \%$ vs $60 \%$; OR, $4.5 ; P=.04)$. The sensitivity and specificity of the MASCC score to predict severe complications among bacteremic patients were $43 \%$ and $86 \%$, respectively.
Predictors of bacteremia in apparently stable patients Univariate analysis was performed to evaluate the risk factors for bacteremia (Table 4). On multivariate analysis (Table 5), the predictors of clinically relevant bacteremia were: temperature $\geq 39^{\circ} \mathrm{C} / 102.2^{\circ} \mathrm{F}(\mathrm{OR}, 3 ; 95 \% \mathrm{CI}$, 1.19-5.40), rigors $(\mathrm{OR}, 2.2 ; 95 \% \mathrm{CI}, 1.05-5.25), \mathrm{ECOG}$ PS (Eastern Cooperative Oncology Group Performance Status) $\geq 2$ (OR, 2.1; 95\% CI, 0.85-5.61), and advanced cancer (OR, 2.5; 95\% CI, 1.26-7.32). Of note, 15 patients $(2 \%)$ who remained afebrile, with sterile blood cultures after 48 hours, were eventually diagnosed with bacteremia.

\section{Discussion}

In recent years, randomized clinical trials have demonstrated that outpatient management for low-risk $\mathrm{FN}$ is both feasible and safe, with selection criteria generally involving the absence of shock, acute organ dysfunction, or specific infections. ${ }^{22}$ The MASCC score is the most validated risk prediction tool to select low-risk patients, but the optimal method for making acceptable risk decisions remains uncertain, ${ }^{23}$ since serious complications occur in $9 \%-15 \%$ of predicted low-risk episodes despite the application of this index. ${ }^{7,24}$ Therefore, it is of great interest among clinicians to identify causes of prognostic

\begin{tabular}{|c|c|c|c|c|}
\hline Characteristic & $\begin{array}{l}\text { No complications, } \\
\quad n=\mathbf{2 8} \\
\text { No. episodes (\%)a }\end{array}$ & $\begin{array}{l}\text { Complications, } \\
\quad n=14 \\
\text { No. episodes (\%)a }\end{array}$ & OR & $P$ value \\
\hline MASCC score $\geq 21$ & $24(86)$ & $8(57)$ & 4.5 & .05 \\
\hline $\begin{array}{l}\text { MASCC variables } \\
\text { Burden of disease } \\
\text { Low } \\
\text { Moderate } \\
\text { Hypotension } \\
\text { COPD } \\
\text { No solid tumor } \\
\text { Dehydration } \\
\text { Inpatient status } \\
\text { Age } \geq 65 \text { y }\end{array}$ & $\begin{array}{l}15(54) \\
13(46) \\
0 \\
5(18) \\
0 \\
8(28) \\
0 \\
12(43)\end{array}$ & $\begin{array}{l}6(43) \\
8(57) \\
0 \\
7(50) \\
0 \\
8(57) \\
0 \\
7(50)\end{array}$ & $\begin{array}{c} \\
\mathrm{na} \\
4.6 \\
\mathrm{na} \\
5 \\
\mathrm{na} \\
1.3\end{array}$ & $\begin{array}{l}\text { na } \\
.03 \\
\text { na } \\
.03 \\
\text { na } \\
\text { ns }\end{array}$ \\
\hline TNM III - IV (advanced cancer) & $19(68)$ & $11(79)$ & 1.7 & ns \\
\hline Male gender & $13(46)$ & $10(71)$ & 2.8 & ns \\
\hline ECOG PS $\geq 2$ & $10(36)$ & $5(36)$ & 1 & ns \\
\hline $\begin{array}{l}\text { Neutrophil count of } \\
<100 \text { cells } / \mathrm{mm}^{3}\end{array}$ & $16(59)$ & $7(50)$ & 0.6 & ns \\
\hline Mucositis $\mathrm{NCl} \geq 2$ & $4(14)$ & $5(36)$ & 3.3 & ns \\
\hline Chronic heart disease & 0 & $2(14)$ & na & ns \\
\hline Lower RTI & $4(14)$ & $6(43)$ & 4.5 & 0.04 \\
\hline \multicolumn{5}{|c|}{$\begin{array}{l}\text { COPD, chronic obstructive pulmonary disease; ECOG PS, Eastern Cooperative Oncology Group Performance Status; G-CSF, granulocyte-colony stimulating factor; } \\
\text { MASCC, Multinational Association of Supportive Care in Cancer; na, not applicable; NCI, National Cancer Institute; ns, not significant; RTI, respiratory tract infection; } \\
\text { OR, odds ratio; TNM, tumor-node metastasis }\end{array}$} \\
\hline
\end{tabular}




\begin{tabular}{|c|c|c|c|c|c|}
\hline Variable & $\begin{array}{c}\text { Bacteremia } \\
(\mathbf{n}=42)^{a}\end{array}$ & $\begin{array}{l}\text { No bacteremia } \\
(n=223)\end{array}$ & OR & $95 \% \mathrm{Cl}$ & P value \\
\hline Age $\geq 65 y$ & $19(45)$ & $59(26)$ & 2.2 & $1.1-4.5$ & .01 \\
\hline Male gender & $23(55)$ & 88 (39) & 1.8 & $0.9-3.6$ & ns \\
\hline Diabetes mellitus & $4(9)$ & $14(6)$ & 1.5 & $0.4-5$ & ns \\
\hline TNM stage III - IV (advanced cancer) & $30(71)$ & $101(45)$ & 3 & $1.4-6.2$ & .02 \\
\hline Chills ${ }^{b}$ & $23(55)$ & $69(31)$ & 2.8 & $1.4-5.5$ & .002 \\
\hline Chemotherapy for advanced disease & $37(88)$ & $145(65)$ & 4 & $1.5-10$ & .003 \\
\hline Central catheter & $8(19)$ & $18(8)$ & 1.9 & $0.7-4.8$ & ns \\
\hline $\mathrm{FN}$ risk category $\geq 20 \%$ & $10(24)$ & $44(20)$ & 1.3 & $0.6-2.9$ & ns \\
\hline Monochemotherapy & $9(21)$ & $26(12)$ & 1.9 & $0.8-4.4$ & ns \\
\hline G-CSF PP & $9(21)$ & $54(24)$ & 0.8 & $0.3-1.8$ & ns \\
\hline Lung cancer & $11(26)$ & $47(21)$ & 1.3 & $0.6-2.8$ & ns \\
\hline Breast cancer & $9(21)$ & $85(38)$ & 0.4 & $0.2-0.9$ & .03 \\
\hline COPD & $12(28)$ & $32(14)$ & 2.3 & $1.1-5.1$ & .02 \\
\hline Chronic heart failure & $2(5)$ & $14(6)$ & 0.7 & $0.1-3.4$ & ns \\
\hline ECOG PS $\geq 2$ & $15(36)$ & $34(15)$ & 3 & $1.4-5.4$ & .002 \\
\hline Albumin $<3 \mathrm{~g} / \mathrm{dL}^{-1}$ at the onset & $16(38)$ & $90(40)$ & 0.9 & $0.4-1.8$ & $\mathrm{~ns}$ \\
\hline Stress-induced hyperglycemia ${ }^{c}$ & $18(43)$ & $65(29)$ & 1.8 & $0.9-3.5$ & .07 \\
\hline Neutrophils $\leq 100$ cells $/ \mathrm{mm}^{-3}$ & $23(55)$ & $99(44)$ & 1.5 & $0.8-3.1$ & ns \\
\hline Monocytes $<200 \mathrm{~mm}^{-3}$ & $31(74)$ & $122(55)$ & 2.3 & $1.1-4.8$ & .02 \\
\hline Hemoglobin $\leq 9 \mathrm{~g} / \mathrm{dl}^{-1}$ & $11(26)$ & $36(16)$ & 1.8 & $0.8-4$ & ns \\
\hline Platelets $<100,000 \mathrm{~mm}^{-3}$ & $26(63)$ & 104 (47) & 1.9 & $0.9-3.9$ & .05 \\
\hline Lymphocytes $<450 \mathrm{~mm}^{-3}$ & $28(67)$ & $110(49)$ & 2 & $1.02-4.1$ & .03 \\
\hline MASCC score, $<21$ & $10(24)$ & $22(10)$ & 2.8 & $1.2-6.5$ & .01 \\
\hline $\begin{array}{l}\text { Burden of disease } \\
\text { Low-asymptomatic } \\
\text { Moderate }\end{array}$ & $\begin{array}{l}18(46) \\
21(54)\end{array}$ & $\begin{array}{l}153(70) \\
65(30)\end{array}$ & 2.6 & $1.3-5.1$ & .03 \\
\hline Upper-respiratory infection & 0 & $22(10)$ & - & - & .03 \\
\hline Lower-respiratory infection & $10(24)$ & $38(17)$ & 1.5 & $0.6-3.3$ & $\mathrm{~ns}$ \\
\hline Enteritis & $3(7)$ & $27(12)$ & 0.5 & $0.1-1.9$ & ns \\
\hline Urinary tract infection & $11(26)$ & $19(8)$ & 3.8 & $1.6-8.7$ & .01 \\
\hline Clinical site of infection & $29(69)$ & $125(56)$ & 1.7 & $0.8-3.5$ & ns \\
\hline Dehydration & $16(38)$ & $48(21)$ & 3.1 & $1.4-6.5$ & .02 \\
\hline Temperature at onset $\geq 39^{\circ} \mathrm{C} / 102.2^{\circ} \mathrm{F}$ & $22(52)$ & $46(21)$ & 4.2 & $2.1-8.4$ & $<.001$ \\
\hline Fever for $\geq 24 \mathrm{~h}$ & $25(59)$ & $86(38)$ & 2.4 & $1.2-4.8$ & .009 \\
\hline Mucositis $\mathrm{NCl}$ grade $\geq 2$ & $9(21)$ & $53(24)$ & 0.8 & $0.3-1.9$ & ns \\
\hline \multicolumn{6}{|c|}{$\begin{array}{l}\text { Cl, confidence interval; COPD, chronic obstructive pulmonary disease; ECOG PS, Eastern Cooperative Oncology Group Performance Status; G-CSF, granulocyte- } \\
\text { colony stimulating factor; MASCC, Multinational Association of Supportive Care in Cancer; NCI, National Cancer Institute; ns, not significant; OR, odds ratio; TNM, } \\
\text { tumor-node-metastasis } \\
\text { opercentages do not total } 100 \text { because of rounding. bUnresected TNM stage III tumors associated with specific anatomic distortions. 'Stress-induced hyperglycemia: } \geq \\
121 \mathrm{mg} / \mathrm{dL}-1 \text { l } \geq 250 \mathrm{mg} / \mathrm{dL}-2 \text { in diabetic patients). }\end{array}$} \\
\hline
\end{tabular}




\begin{tabular}{|c|c|c|c|c|}
\hline Variable & Coefficient & OR & $95 \% \mathrm{Cl}^{\circ}$ & $P$ value \\
\hline Temperature $\geq 39^{\circ} \mathrm{C} / 102.2^{\circ} \mathrm{F}$ & 1.17 & 3.2 & $1.19-5.40$ & .002 \\
\hline Rigors & 0.84 & 2.3 & $1.05-5.25$ & .02 \\
\hline$E C O G P S \geq 2$ & 0.80 & 2.2 & $0.85-5.61$ & .07 \\
\hline TNM stage IIIb-IV (advanced cancer) & 0.99 & 2.7 & $1.26-7.32$ & .01 \\
\hline
\end{tabular}

uncertainty in order to reduce the rate of misclassification and optimize individualized management.

Documented bacteremia has been reported to be around $10 \%$ in neutropenic patients with solid tumors. ${ }^{8,25,26}$ Several studies have reported higher attributable mortality rates in neutropenic patients with bacteremia, ${ }^{4,5}$ and this should be taken into account in patients who are discharged home. Unfortunately, careful clinical assessment does not necessarily prevent the discharge of some patients with unsuspected bloodstream infections. Although some of these cases might respond well to oral treatment, several studies on outpatient management have shown bacteremia to be a cause of hospital readmission and severe complications. ${ }^{27,28}$ Microbial sensitivity to oral antibiotics is another major concern in bacteremic patients. For example, during the study period, only $38 \%$ of the $E$ coli isolates were sensitive to ciprofloxacin.

Therefore, it remains an area of interest for physicians to determine the causes and risk factors of bacteremia in otherwise low-risk episodes. We have found that a simple definition of clinical stability, based on the exclusion of the most severe infections, ${ }^{20}$ is associated with a low rate of bacteremia ( $6 \%$ of episodes). Nevertheless, bacteremic status showed substantial effects on patient prognosis, yielding a high rate of complications $(33 \%, 14$ of 42 episodes) and infection-associated mortality (7\%, 3 of 42 episodes), thereby defining this group as a high-risk population. Moreover, bacteremia was also associated with other adverse outcomes such as longer hospital stay and treatment failure.

Peripheral blood cultures taken in the emergency department are not available at the time of initial assessment. Because failure to suspect possible bacteremia may lead to undertreatment in some cases, it is also important to determine whether bacteremia may be predicted in clinically stable neutropenic outpatients. Previous reports have shown that bacteremia is particularly difficult to predict in this setting, with a low positive predictive value in a validation set for a prediction model. ${ }^{29}$ We have identified 4 risk factors to aid in the prediction of bacteremia in ASPs: temperature $\geq 39^{\circ} \mathrm{C} / 102.2^{\circ} \mathrm{F}$, rig- ors, ECOG PS of $\geq 2$, and advanced cancer. Although these variables should be confirmed in further validation studies, they may be of use in neutropenic bacteremia. High-grade fever has previously been associated with bacteremia in low-risk FN episodes, ${ }^{26}$ with the rate of bacteremia generally being correlated with the magnitude of fever. ${ }^{29-31}$ Rigors have also been related to bacteremia in both neutropenic, ${ }^{32,33}$ and non-neutropenic patients. ${ }^{34}$ The performance status has been proposed as a prognostic factor in low-risk $\mathrm{FN},{ }^{24}$ and both immunosuppression and the anatomical distortion associated with advanced cancer are risk factors predisposing serious infections.

With respect to the performance of the MASCC score, Paesmans and colleagues have reported that the integration of the bacteremic status did not increase the accuracy of the MASCC model to identify patients at low-risk of life-threatening complications. ${ }^{8}$ One way to interpret that observation is that the MASCC score is effective in identifying low-risk bacteremic episodes. This effectiveness has also been attributed to the Talcott classification. ${ }^{25}$ However, none of these previous series were stratified according to the clinical presentation, thus, the prognostic role in specific populations, such as clinically stable patients, remains uncertain. Although the addition of bacteremic status to the MASCC score did not improve its discriminatory ability, one of the elements of concern that arise from our data is that $68 \%$ of bacteremic patients had a MASCC score of $\geq 21$, whereas $57 \%$ of the complications occurred among predicted low-risk episodes (Table 3).

Beyond the necessity for further confirmation, the potential implications in clinical practice or possible future directions for research that could emerge from these data are conditioned by the fact that all patients from this cohort were managed as inpatients. As a result, it is unlikely that our conclusions could be used to select cases for hospital discharge but actually to identify those apparently healthier patients who should never be sent home because they are at risk of developing serious infections. Conversely, given the low probability $(2 \%)$ of bac- 
teremia in patients who are afebrile with a provisional sterile culture after 48 hours, a strategy of early rather than immediate discharge could be recommended as long as the bacteremic status remains of concern in specific patients, despite being determined to be clinically stable at first presentation.

With respect to the criteria for discrimination between true bacteremia and contamination, all cases fulfilled the CDC-NHSN definition of clinically significant bloodstream infection. ${ }^{21}$ Therefore, we excluded 2 cases in which CoNS was isolated in only 1 blood sample. However, 4 other isolates of CoNS were identified as potentially true bacteremias, with central-line infection in 3 patients and cellulitis in the fourth. These results are in accordance with García-Vázquez and colleagues, who have recently analyzed this issue and found that cancer, chemotherapy, neutropenic sepsis and previous immunosuppression are associated with clinically significant bacteremia by CoNS. ${ }^{35}$ Isolations of Staphylococcus hominis and Bacteroides uniformis were also identified as true bacteremias, because patients had a significant cutaneous barrier disruption and advanced colorectal cancer, respectively.

In conclusion, bacteremia is a potential source of prognostic uncertainty in patients with FN otherwise considered clinically stable. Although accurate prediction will continue to be difficult in the future, clinical suspicion of bloodstream infections can be useful in neutropenic patients to determine the optimal time of hospital discharge.

\section{References}

1. Gatell JM, Trilla A, Latorre X, et al. Nosocomial bacteremia in a large Spanish teaching hospital: analysis of factors influencing prognosis. Clin Infect Dis. 1988;10:203-210.

2. Kuderer NM, Dale DC, Crawford J, et al. Mortality, morbidity, and cost associated with febrile neutropenia in adult cancer patients. Cancer. 2006;106:2258-2266.

3. Paganini HR, Aguirre C, Puppa G, et al. A prospective, multicentric scoring system to predict mortality in febrile neutropenic children with cancer. Cancer. 2007;109:2572-2579.

4. Micek ST, Lloyd AE, Ritchie DJ, et al. Pseudomonas aeruginosa bloodstream infection: importance of appropriate initial antimicrobial treatment. Antimicrob Agents Chemother. 2005;49:13061311.

5. Bodey GP. Pseudomonas bacteremia. Retrospective analysis of 410 episodes. Arch Intern Med. 1985;145:1621-1629.

6. Freifeld AG, Bow EJ, Sepkowitz KA, et al. Infectious Diseases Society of America. Clinical practice guideline for the use of antimicrobial agents in neutropenic patients with cancer: 2010 update by the infectious diseases society of America. Clin Infect Dis. 2011;52:e56-93.

7. Klastersky J, Paesmans M, Rubenstein EB, et al. The Multinational Association for Supportive Care in Cancer risk index: a multinational scoring system for identifying low-risk febrile neutropenic cancer patients. J Clin Oncol. 2000;18:3038-3051.

8. Paesmans M, Klastersky J, Maertens J, et al. Predicting febrile neutropenic patients at low risk using the MASCC score: does bacte- remia matter? Support Care Cancer. 2011;19:1001-1008.

9. Klastersky J, Ameye L, Maertens J, et al. Bacteraemia in febrile neutropenic cancer patients. Int J Antimicrob Agents. 2007;30:S51-S59.

10. Coburn B, Morris AM, Tomlinson G, et al. Does this adult patient with suspected bacteremia require blood cultures? JAMA. 2012;308:502-511

11. Wisplinghoff $\mathrm{H}$, Seifert $\mathrm{H}$, Wenzel RP, et al. Current trends in the epidemiology of nosocomial bloodstream infections in patients with hematological malignancies and solid neoplasms in hospitals in the United States. Clin Infect Dis. 2003;36:1103-1110.

12. Klastersky J. Management of fever in neutropenic patients with different risks of complications. Clin Infect Dis. 2004;39:32-37.

13. Uys A, Rapoport BL, Anderson R. Febrile neutropenia: a prospective study to validate the Multinational Association of Supportive Care of Cancer (MASCC) risk-index score. Support Care Cancer. 2004; $12: 555-560$

14. Weinstein MP. Current blood culture methods and systems: clinical concepts, technology, and interpretation of results. Clin Infect Dis. 1996;23:40-46.

15. Sanz MA, Lopez J, Lahuerta JJ, et al. Cefepime plus amikacin versus piperacillin-tazobactam plus amikacin for initial antibiotic therapy in haematology patients with febrile neutropenia: results of an open, randomized, multicentre trial. J Antimicrob Chemother. 2002;50:79-88.

16. Wildi K, Tschudin-Sutter S, Dell-Kuster S, et al. Factors associated with positive blood cultures in outpatients with suspected bacteremia. Eur J Clin Microbiol Infect Dis. 2011;30:1615-1619.

17. Gardembas-Pain M, Desablens B, Sensebe L, et al. Home treatment of febrile neutropenia: an empirical oral antibiotic regimen. Ann Oncol. 1991;2:485-487.

18. Rubenstein EB, Rolston K, Benjamin RS, et al . Outpatient treatment of febrile episodes in low-risk neutropenic patients with can cer. Cancer. 1993;71:3640-3646.

19. Hughes WT, Armstrong D, Bodey GP, et al. 2002 guidelines for the use of antimicrobial agents in neutropenic patients with cancer. Clin Infect Dis. 2002;34:730-751.

20. Carmona-Bayonas A, Gómez J, González-Billalabeitia E, et al. Prognostic evaluation of febrile neutropenia in apparently stable adult cancer patients. Br J Cancer. 2011;105:612-617.

21. Horan TC, Andrus M, Dudeck MA. CDC/NHSN surveillance definition of health care-associated infection and criteria for specific types of infections in the acute care setting. Am J infect Control. 2008;36:309-332.

22. Teuffel O, Ethier MC, Alibhai SM, at al. Outpatient management of cancer patients with febrile neutropenia: a systematic review and meta-analysis. Ann Oncol. 2011;22:2358-2365

23. Flowers CR, Seidenfeld J, Bow EJ, et al. Antimicrobial prophylaxis and outpatient management of fever and neutropenia in adults treated for malignancy: American Society of Clinical Oncology clinical practice guideline. J Clin Oncol. 2013;31:794-810.

24. Klastersky J, Paesmans M, Georgala A. Outpatient oral antibiotics for febrile neutropenic cancer patients using a score predictive for complications. J Clin Oncol. 2006;24:4129-4134.

25. Talcott JA, Siegel RD, Finberg R, Goldman L. Risk assessment in cancer patients with fever and neutropenia: a prospective, two-center validation of a prediction rule. J Clin Oncol. 1992;10:316-322.

26. Ha YE, Song JH, Kang WK, Peck KR, et al. Clinical factors predicting bacteremia in low-risk febrile neutropenia after anti-cancer chemotherapy. Support Care Cancer. 2011;19:1761-1767.

27. Gardembas-Pain M, Desablens B, Sensebe L, et al. Home treatment of febrile neutropenia: an empirical oral antibiotic regimen. Ann Oncol. 1991;2:485-487.

28. Rubenstein EB, Rolston K, Benjamin RS, et al. Outpatient treatment of febrile episodes in low-risk neutropenic patients with cancer. Cancer. 1993;71:3640-3646.

29. Viscoli C, Bruzzi P, Castagnola E et al. Factors associated with bacteremia in febrile, granulocytopenic cancer patients. Eur J Cancer. 1994;30:430-437.

30. Rackoff WR, Gonin R, Robinson C, et al. Predicting the risk of bacteremia in childen with fever and neutropenia. J Clin Oncol. 1996;14:919-924. 


\section{Original Report}

31. Baorto EP, Aquino VM, Mullen CA, et al. Clinical parameters associated with low bacteremia risk in 1100 pediatric oncology patients with fever and neutropenia. Cancer. 2001;92:909-913.

32. Agyeman P, Aebi C, Hirt A, et al. Predicting bacteremia in children with cancer and fever in chemotherapy-induced neutropenia: results of the prospective multicenter SPOG 2003 FN study. Pediatr Infect Dis J. 2011;30:e114-9.

33. Spasova MI, Grudeva-Popova JG, Kostyanev SS, et al. Risk index score for bacteremia in febrile neutropenic episodes in children with malignancies. J BUON. 2009;14:411-418.

34. Lee CC, Wu CJ, Chi CH, et al. Prediction of community-onset bacteremia among febrile adults visiting an emergency department: rigor matters. Diagn Microbiol Infect Dis. 2012;73:168 173.

35. García-Vázquez E, Fernández-Rufete A, Hernández-Torres A, et al. When is coagulase-negative Staphylococcus bacteraemia clinically significant? Scand J Infect Dis. 2013;45:664-671. 